\title{
A Comparison of Bispectral Index and Entropy During Sevoflurane Anesthesia Induction in Children with and without Diplegic Cerebral Palsy
}

\author{
Young Sung Kim ${ }^{\circledR}$, Young Ju Won, Hyerim Jeong, Byung Gun Lim ${ }^{\circledR}$, Myoung Hoon Kong and \\ Il Ok Lee *(1) \\ Department of Anesthesiology and Pain Medicine, Korea University Guro Hospital, Seoul 08308, Korea; \\ stelios@naver.com (Y.S.K.); moma2@naver.com (Y.J.W.); faveur1117@gmail.com (H.J.); \\ bglim9205@korea.ac.kr (B.G.L.); kong0331@korea.ac.kr (M.H.K.) \\ * Correspondence: iloklee@korea.ac.kr; Tel.: +82-2-2626-1437
}

Received: 29 March 2019; Accepted: 14 May 2019; Published: 15 May 2019

\begin{abstract}
Background: This study compared the correlation of bispectral index (BIS) or entropy with different sevoflurane concentrations between children with and without cerebral palsy (CP) during induction. Methods: For eighty-two children (40 CP and 42 non-CP children), anesthesia was induced with sevoflurane. BIS and entropy (response entropy and state entropy (RE and SE)) were recorded before and after the induction of anesthesia at end-tidal sevoflurane concentrations of 1-3 vol\%. The sedation status was assessed using an Observer's Assessment of Alertness/Sedation scale. The ability to predict awareness was estimated using the area under the receiver-operator characteristic curve (AUC) analysis. Results: RE, SE and BIS values decreased continuously over the observed concentration range of sevoflurane in both groups. The $\mathrm{SE}$ values while awake and the RE, $\mathrm{SE}, \mathrm{BIS}$ values at $3 \mathrm{vol} \%$ sevoflurane were lower in children with $\mathrm{CP}$ than in those without $\mathrm{CP}$. The AUC of the BIS was significantly better than RE or SE in children without CP. The AUC of the BIS was not significantly higher than that of the RE or SE in children with CP. Conclusion: BIS seems better correlated than entropy with the clinical state of loss of response in children without $\mathrm{CP}$, but not in those with $\mathrm{CP}$.
\end{abstract}

Keywords: cerebral palsy; consciousness monitors; electroencephalography; sevoflurane

\section{Introduction}

Monitoring the depth of anesthesia (DOA) has proven difficult. The two monitors which are commercially available, the bispectral index (BIS) monitor, providing the BIS values, and the entropy, providing the response entropy (RE) and state entropy (SE) values [1], have disturbingly large inter-individual variation and lack of linearity in dose-response [2]. A recent study suggest that the prediction probabilities $\left(P_{\mathrm{K}}\right)$ for BIS, RE and SE were similar in pediatric patients [3]. A previous study showed that children with cerebral palsy require less propofol to reach a BIS of 35-45 than healthy children [4]. Although, Choudhry and Brenn [5] showed the reliability of BIS in children with cerebral palsy, few studies have analyzed the effects of entropy in cerebral palsy. This study aimed to compare the validity between the BIS and entropy in cerebral palsy.

We hypothesized that there would be no difference in BIS or entropy values between two groups of children with or without cerebral palsy. Based on the outcome of a previous study, the correlation of BIS or entropy values with different sevoflurane concentrations during induction was set as the primary endpoint. Other observations (incidence of excitement during sevoflurane induction, end-tidal sevoflurane concentration at loss of response, and different values of BIS or entropy at loss of response) 
were set as secondary endpoints. The predictive power in discriminating the loss of response state from the awake state was the tertiary endpoint. To test this hypothesis, we compared the correlation of BIS or entropy values with different sevoflurane concentrations during induction between children without cerebral palsy and those with diplegic cerebral palsy.

\section{Materials and Methods}

The study was approved by the Institutional Review Board of Korea University Guro Hospital (GR0832-002). The study objective and methods were clearly explained to all parents/guardians and written informed consent was obtained. We prospectively studied children who had normal intelligence with or without diplegic cerebral palsy with American Society of Anesthesiologists physical status I or II and scheduled for elective orthopedic surgery. The exclusion criteria were a history of anti-epileptic medication; mental retardation; cardiac, pulmonary, or renal disease; and contraindication for an inhalational induction. Due to the low incidence of cerebral palsy, we included all cerebral palsy (CP) patients who visited during the experimental period. Each non- $\mathrm{CP}$ patient who visited next to a $\mathrm{CP}$ patient was included for matching the 1:1 number of patients.

All patients were sedated $30 \mathrm{~min}$ before anaesthesia induction with midazolam $0.5 \mathrm{mg} / \mathrm{kg}$ intramuscularly, and underwent electrocardiography, pulse oximetry, and non-invasive blood pressure monitoring. BIS values were recorded on an aspect medical system monitor (model A2000; Natick, MA, USA; smoothing time, $15 \mathrm{~s}$ ) using commercially available BIS-XP sensor strips (Aspect Medical Systems, Newton, MA, USA). An entropy sensor (M-entropy plug-in Module S/5 ${ }^{\mathrm{TM}}$; Datex-Ohmeda Division, Instrumentarium Corp., Helsinki, Finland) was used for collecting entropy values. After sterilizing the forehead skin using alcohol swabs, the sensors were placed in close proximity. The temporal electrodes for BIS and entropy measurements were placed to the right and left, respectively. Two sensors were placed in the immediate vicinity of each other: the entropy sensor lower on the forehead and the BIS sensor higher. The BIS uses a 100-point index score, wherein values close to 100 indicate the awake state, and values close to 0 indicate very deep general anaesthesia. Entropy values were in the range of 0-91 for state entropy, or 0-100 for response entropy.

Anesthesia was induced using sevoflurane inhalation alone via a tight-fitting facemask and head strap held by a single experienced pediatric anesthesiologist. We confirmed that air leakage from the margin of the facemask was minimal when positive or negative airway pressure was applied. The facemask was connected to a semi-closed circle system with a fresh gas flow of $6 \mathrm{~L} / \mathrm{min}$ of $100 \%$ oxygen. The end-tidal sevoflurane concentrations and carbon dioxide concentrations were continuously monitored using the $\mathrm{S} / 5^{\mathrm{TM}}$ compact monitor. Baseline measurements were made after resting $5 \mathrm{~min}$ in the supine position; thereafter, sevoflurane was administered continuously and its concentration was increased once every $5 \mathrm{~min}$ in increments of $1 \%$ to achieve a steady state of end-tidal sevoflurane concentrations of $1 \%, 2 \%$, and $3 \%$. We maintained partial pressure of $\mathrm{CO}_{2}\left(\mathrm{EtCO}_{2}\right)$ of $35-40 \mathrm{mmHg}$. When respiratory depression or $\mathrm{CO}_{2}$ retention had presented, mask ventilation was assisted to the patients. Loss of consciousness (LOC) was determined as the loss of response to loud verbal commands (LOR) by name and shaking on the shoulder every $10 \mathrm{~s}$. The Observer's Assessment of Alertness/Sedation rating (OAA/S) scale was used: score $5=$ awake and responds readily to the name spoken in a normal tone; $4=$ lethargic response to the name in a normal tone; $3=$ responds only after the name is called loudly and repeatedly; 2 = responds only after the name is called loudly and after mild shaking; and $1=$ does not respond when the name is called and mild shaking. All assessments of sedation level were performed by one investigator (Lee I.O.) to minimize inter-observer variability. At the end of the study period, measurements were discontinued, and anesthesia was deepened using an appropriate dose of propofol and neuromuscular blocking agents; thereafter, the patients were intubated for surgery. All values from the anesthetic monitoring (including BIS and entropy scores) were recorded every $5 \mathrm{~s}$ to a personal computer.

Preliminary data analysis suggested that excitement behaviors or loss of responsiveness would occur between $1 \%$ and $2 \%$ end-tidal sevoflurane concentrations. Additional observations were added 
for $1.5 \%$ to estimate more clearly the excitatory effect of sevoflurane in patients showing excitement during the study period. These children were excluded from the following DOA assessments. Other children without excitement behavior were also included in the study.

A power analysis suggested that a minimum sample size of 40 patients for each group would be required with a significance level of $5 \%$ to achieve a power of $80 \%$. It was calculated from SE values at $3 \%$ sevoflurane in preliminary data (effect size $d=0.636$ calculated from $38.6 \pm 14.3$ of SE in CP versus $46.3 \pm 9.4$ of $\mathrm{SE}$ in non-CP patients). To allow for an exclusion rate, the study population was prospectively set at 90 patients.

Statistical analysis was performed using Sigma Stat for window v3.01 (2004 Systat software, Inc, $\mathrm{GmbH}$, Germany). The analyzed data were tested for normality using the Kolmogorov-Smirnov test. A parametric or non-parametric analysis was performed depending upon the results of the Kolmogorov-Smirnov analysis. Data expressed as mean \pm SD were compared using independent $t$-tests or Mann-Whitney U tests. Additionally, data expressed as the number of patients were compared using chi-square analysis or Fisher's exact test, where appropriate. Statistical significance was defined as $p<0.05$; all data are represented as mean (standard deviation) or median (interquartile range).

Receiver-operator characteristic (ROC) analysis and comparison of ROC curves were performed using Sigma Plot for Windows v11.0 (2008 Systat Software, Inc). ROC curve analysis is a technique used to assess the overall ability of a "test" to differentiate between normal and abnormal populations of results. The "tests" investigated in this study were BIS, RE and SE. Data were sampled from representing responsiveness present, that is, awake and up to LOR during sevoflurane induction.

\section{Results}

Forty children with cerebral palsy (Group CP) and 42 without cerebral palsy (Group C) were finally enrolled. Eight patients (five for Group $\mathrm{CP}$ and three for Group $\mathrm{C}$ ) were excluded from this study because of the lack of informed consent. The most type of $\mathrm{CP}$ was spastic (31 patients; $77.5 \%$ ). Seven patients $(17.5 \%)$ had dyskinetic CP and the other two patients $(5 \%)$ had ataxic CP. In the Group $\mathrm{C}$, there were two patients with achondroplasia. The other 40 patients were of American Society of Anesthesiologists' physical status classification 1 (ASA 1 refers to a normal healthy patient) and had no medical history. Their demographic data showed no significant differences in age, sex, weight and height (Table 1).

Table 1. Demographic data.

\begin{tabular}{ccc}
\hline & Group C $(\mathbf{n}=\mathbf{4 2})$ & Group CP $(\mathbf{n}=\mathbf{4 0 )}$ \\
\hline Age $($ year $)$ & $10.2 \pm 2.9$ & $11.1 \pm 2.0$ \\
\hline Sex $(\mathrm{M} / \mathrm{F})$ & $20 / 22$ & $14 / 26$ \\
\hline Weight $(\mathrm{kg})$ & $25.6 \pm 10.8$ & $23.0 \pm 9.4$ \\
\hline Height $(\mathrm{cm})$ & $116.0 \pm 18.7$ & $118.6 \pm 18.4$ \\
\hline
\end{tabular}

Values are mean \pm SD or number of patients. Group C: Children without cerebral palsy, Group CP: Children with diplegic cerebral palsy.

The baseline value of SE was significantly lower in Group CP than in Group C. During sevoflurane induction, excitement was noted in 26 children $(62 \%)$ in Group C and $24(60 \%)$ in Group CP. The end-tidal sevoflurane concentration at the beginning of excitement was significantly higher in Group $\mathrm{C}$ than in Group CP. However, the end-tidal sevoflurane concentration at LOR showed no significant intergroup difference. The RE, SE, and BIS values at $3 \%$ end-tidal sevoflurane concentration were significantly lower in Group CP than in Group C (Table 2). 
Table 2. RE, SE, BIS values and end-tidal (ET) sevoflurane concentrations during the induction periods.

\begin{tabular}{cccc}
\hline & & Group C (n = 42) & Group CP (n = 40) \\
\hline \multirow{2}{*}{ Awake state } & RE & $97.5 \pm 1.7$ & $96.8 \pm 2.0$ \\
\cline { 2 - 4 } & SE & $89.5 \pm 2.9$ & $86.9 \pm 2.0 *$ \\
\hline ET Sevoflurane (vol\%) (beginning excitement) & $1.6 \pm 0.1$ & $93.1 \pm 5.7$ \\
\hline ET Sevoflurane (vol\%) (unresponsiveness) & $1.7 \pm 0.4$ & $1.3 \pm 0.1{ }^{*}$ \\
\hline \multirow{2}{*}{$\begin{array}{c}\text { At 3 vol\% of ET } \\
\text { Sevoflurane }\end{array}$} & RE & $53.3 \pm 11.1$ & $39.1 \pm 0.3$ \\
\cline { 2 - 4 } & SE & $47.7 \pm 9.9$ & $34.8 \pm 10.2 *$ \\
\hline
\end{tabular}

Values are mean \pm SD. Group C: Children without cerebral palsy, Group CP: Children with spastic diplegic cerebral palsy, RE: Response entropy, SE: State entropy, BIS: Bispectral index; ${ }^{*} p<0.05$ compared with Group C.

The OAA/S scale abruptly decreased within ten minutes in the both groups. The OAA/S values at 3, 4, 5, 6 min in Group CP were significantly lower than those in Group C $(\mathrm{P}=0.011,0.002,0.021$ and 0.039 , respectively) (Figure 1).

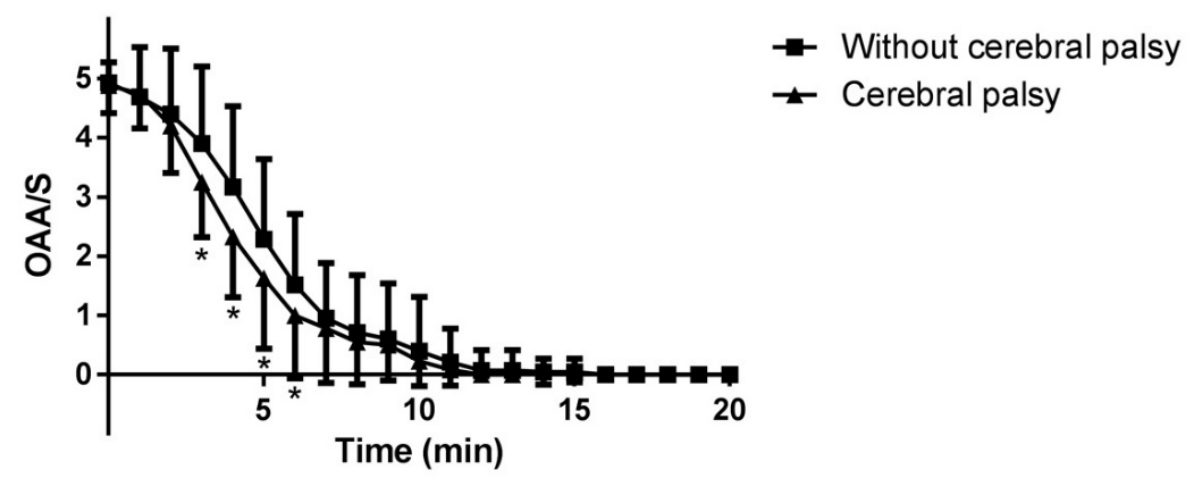

Figure 1. The change of Observer's Assessment of Alertness/Sedation rating (OAA/S) scale during the induction period. Data are shown as mean \pm SD. ${ }^{*} p<0.05$ compared with without cerebral palsy.

Plotting the end-tidal sevoflurane concentration against entropy and BIS values revealed a hysteresis in both groups (Figure 2A-C).

The areas under the ROC curve (AUCs) of both entropy values were significantly higher in Group $\mathrm{CP}$ than in Group C $(p=0.04)$. The intergroup difference between the AUC of BIS values was not statistically significant. The AUC of the BIS was significantly higher than that of response entropy $(p=0.007)$ or state entropy $(p=0.001)$ in Group C (Table 3; Figure 3A,B).

Table 3. Area under the curve (AUC) for responsiveness present.

\begin{tabular}{ccc}
\hline & \multicolumn{2}{c}{ AUC } \\
\cline { 2 - 3 } & Group C $(\mathbf{n}=\mathbf{4 2})$ & Group CP $(\mathbf{n}=\mathbf{4 0 )}$ \\
\hline RE & $0.81 \pm 0.03$ & $0.89 \pm 0.02 *$ \\
\hline SE & $0.79 \pm 0.03$ & $0.90 \pm 0.02 *$ \\
\hline BIS & $0.87 \pm 0.03^{+, \ddagger}$ & $0.90 \pm 0.02$ \\
\hline
\end{tabular}

Values are mean \pm SD. Group C: Children without cerebral palsy, Group CP: Children with spastic diplegic cerebral palsy, RE: Response entropy, SE: State entropy, BIS: Bispectral index; ${ }^{*} p<0.05$ compared with Group $\mathrm{C}^{+} p<0.05$ compared with RE, $\neq p<0.05$ compared with SE. 
A
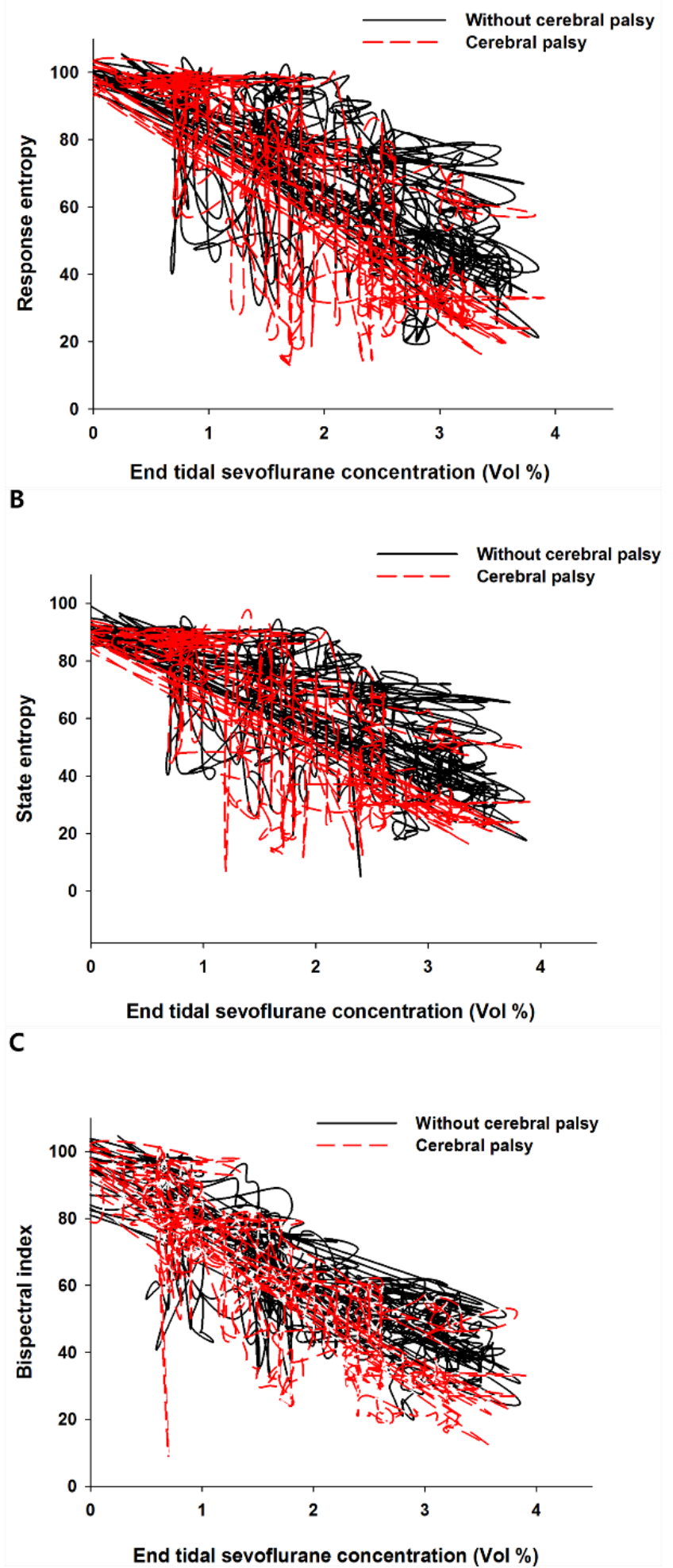

Figure 2. Anesthetic depth and end-tidal sevoflurane concentration during the induction period. This figure shows hysteresis on (A) response entropy, (B) state entropy, or (C) bispectral index with regard to end-tidal sevoflurane concentration. The red dotted lines refer the children with cerebral palsy; the black solid lines refer to the children without cerebral palsy. 
A

ROC Curves (without cerebral palsy)

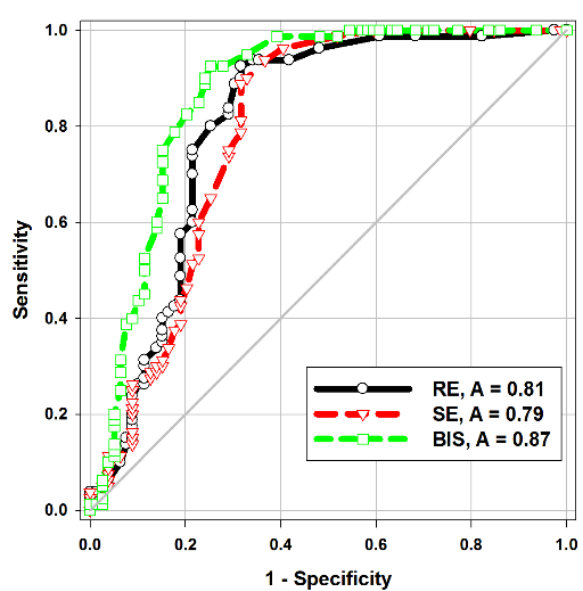

B

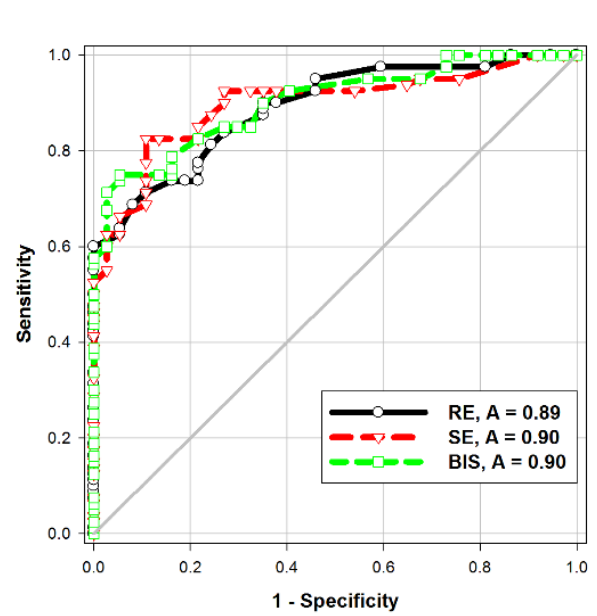

Figure 3. Receiver-operating characteristic (ROC) curves to demonstrate the ability of response entropy (RE), state entropy (SE), and bispectral index (BIS) to predict unresponsiveness in (A) children without cerebral palsy or $(\mathbf{B})$ children with cerebral palsy.

The cut-off values to discriminate whether LOR was presented or not were 91.5 for RE, 84.5 for SE, and 75.5 for BIS in Group C; the corresponding values in Group CP were 75.5, 73, and 65.5, respectively (Table 3; Figure 4A,B).
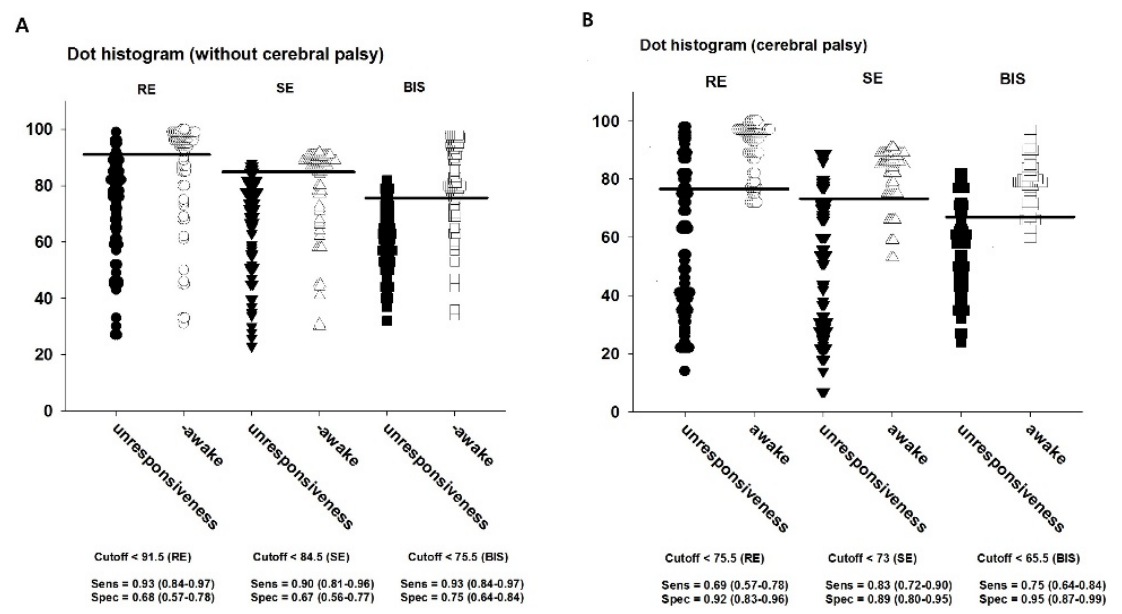

Figure 4. Dot histogram representation of response entropy (RE), state entropy (SE) and bispectral index (BIS) compared with unresponsiveness or awake in the (A) children without cerebral palsy or (B) children with cerebral palsy. Data are shown as median \pm interquartile range. The horizontal lines and the tables to the bottom of the graph represent the cut-off values for RE, SE, and BIS, and the corresponding sensitivity/specificity.

\section{Discussion}

In this study, we compared the BIS and entropy in children with cerebral palsy. The baseline state entropy was significantly lower in children with cerebral palsy than in those without, before anesthetic induction. Cerebral palsy develops from an injury to the developing brain, and has features of motor impairments stemming from non-progressive brain malfunction early in life [6,7]. Therefore, EEG and structural cranial abnormalities is not rare in epileptic CP [8]. Moreover, even children with seizure-free cerebral palsy show electroencephalographic (EEG) abnormalities including asynchronous slow wave, epileptiform activity, and hypsarrhythmia [9]. This reflects heterogeneity of the neural 
generator in the underlying disease process. In the awake state, state entropy was significantly lower in children with cerebral palsy than in those without, while response entropy was not. This can be explained by previous findings [5] showing that non-sedated children with cerebral palsy had high muscle tone with a strong electromyographic (EMG) signal. State entropy reflects only the hypnotic level from EEG, but response entropy includes EEG and forehead EMG components. Even if the children with cerebral palsy received premedication in this study, response entropy could reflect both EEG and EMG components throughout the study period.

Choudhry and Brenn [5] have reported a similar pattern of BIS change in children with quadriplegic cerebral palsy. However, they reported lower absolute BIS values in children with cerebral palsy than in normal children while awake and at different sevoflurane concentrations. Costa et al. [10] also observed lower baseline BIS values and a slower return to the conscious state in children with cerebral palsy than in the control group during recovery of anaesthesia. In our study, BIS values in the awake state were similar between children with and without cerebral palsy. This discordance in results may be attributed to a difference in the type of cerebral palsy, which was diplegic and less severe in our study.

The BIS and entropy values at 3\% end-tidal sevoflurane concentration was significantly lower in children with cerebral palsy than in those without, as seen in a previous study [5]. Moreover, the excitement events occurred earlier in children with cerebral palsy, despite the lower end-tidal sevoflurane concentration. These findings suggest children with cerebral palsy are susceptible to anesthesia [4,11]. Excitement during sevoflurane induction has been previously reported [12,13], while its cause remains unknown, it may be similar to the excitement phase with diethyl ether induction. The incidence of excitement was 35-38\%, and excitement at induction occurred more frequently in children when sevoflurane was used with oxygen alone rather than when combined with nitrous oxide and oxygen [12]. In our study, excitement occurred more frequently $(60 \%$ in children with cerebral palsy and $62 \%$ in those without) than previously reported. We performed very slow induction (approximately $20 \mathrm{~min}$ to reach 3\% end-tidal sevoflurane concentration) with oxygen without nitrous oxide, and think this may have elicited more excitement.

In our study, the AUC of the BIS at LOR was higher than that of response entropy or state entropy in children without cerebral palsy. This finding was also different from that of other prior studies on adults $[14,15]$, which showed that at the point of LOC, the entropy and BIS systems did not differ significantly, suggesting that the BIS and entropy monitoring capacities on the level of sedation are similar [14]. Higher entropy values for deep sedation and surgical anesthesia were reported in younger children (3-6 years) than in older children [16]. In a recent study on adults, state entropy showed more false classification than did the BIS in the transition state from consciousness to unconsciousness, especially in sevoflurane anesthesia rather than propofol anesthesia [17]. This is because EEG signal analysis shows that the high-frequency signal and eye blink cause errors in the degree of consciousness. State entropy may be influenced more by the delta band than is the BIS, and delta band power was relatively lower in cases misclassified using raw EEG signals.

Unlike in the control group, the children with cerebral palsy in our study showed no difference in the AUCs of the BIS and entropy. Kułak and Sobaniec [18] showed a significant decrease of power alpha at occipital derivations, significant increase of theta power and delta bands, and significant decrease of interhemispheric coherence values in children with spastic diplegia. As mentioned above [17], the delta band has a greater influence on state entropy than on the BIS. Therefore, we assumed that entropy values disagreed with clinical assessment in the normal population, which has a relatively lower delta band; however, in children with diplegic cerebral palsy, this could be offset by the increased delta band.

Our study has several limitations. First, CP types in the study were not homogeneous. Although the most type was spastic, the other types, including dyskinetic and ataxic $\mathrm{CP}$, may influence the results and make the interpretation difficult. However, since all the children had not taken anti-epileptic medications, the effect might not be significant. Second, the algorithms for BIS and spectral entropy calculation are sophisticated and the precise BIS algorithm is not available in the public domain [19]. Therefore, we cannot precisely explain the theoretic mechanisms underlying our results. Last, during 
sevoflurane induction, only the LOR was recorded as the patients experienced a state of excitement, making complex assessment impossible.

Despite these limitations, our results provide meaningful information in interpreting the BIS and entropy in children with cerebral palsy, in whom entropy may help distinguish consciousness from unconsciousness as well as the BIS. Considering that entropy describes the irregularity, complexity, or unpredictability of the EEG [20] and assuming that BIS reflects the EEG characteristics of the general population (see the Appendix A), entropy may be useful for $\mathrm{CP}$ patients. Furthermore, looking to exhibiting hysteresis, both BIS and entropy can be said to suggest that the limits of the EEG signal processing for real-time evaluation of consciousness [21].

In conclusion, the absolute state entropy values obtained while awake, as well as the response entropy, state entropy, and BIS at 3\% end-tidal sevoflurane concentration are lower in children with cerebral palsy than in those without. The BIS is better correlated than entropy at the point of LOR during sevoflurane induction in children without cerebral palsy, but not in children with cerebral palsy. A different pattern of background brain activity in children with cerebral palsy may influence entropy more than the BIS. Further evaluation of the correlation between brain activity in cerebral palsy and DOA monitoring is worthwhile, utilizing not only EEG but also other modalities.

Author Contributions: Conceptualization, B.G.L., M.H.K., and I.O.L.; methodology, B.G.L., M.H.K., and I.O.L.; software, Y.S.K., Y.J.W., and H.J.; validation, Y.S.K., Y.J.W., and H.J.; formal analysis, Y.S.K., Y.J.W., and H.J.; investigation, Y.S.K., Y.J.W., and H.J.; resources, B.G.L., M.H.K., and I.O.L.; data curation, B.G.L., M.H.K., and I.O.L.; writing—original draft preparation, Y.S.K.; writing—review and editing, B.G.L., M.H.K., and I.O.L.; visualization, Y.S.K., Y.J.W., and H.J.; supervision, B.G.L., M.H.K., and I.O.L.; project administration, B.G.L., and I.O.L.; funding acquisition, N/A.

Funding: This research received no external funding.

Conflicts of Interest: The authors declare no conflict of interest.

\section{Appendix A. Brief Explanations for BIS and Entropy Algorithms}

Fourier's theorem revealed that all periodic functions can be represented as the sum of simple sine waves. Therefore, any EEG signal can be disassembled to many sine waves by their own frequencies (Fourier transform). Each sine wave distracted by raw EEG may be described by three parameters: amplitude, frequency, and phase angle. Each frequency has power, which individually contributes to the entire wave (amplitude is one half the peak-to-peak voltage; frequency is number of complete cycles per second; and phase angle is the way to describe the starting point of the wave form.) Power spectral analysis represents relative power in each frequency domain. In this way, EEG may convert from time domain to frequency domain. BIS and entropy are widely used complex EEG parameters to quantify anesthetic depth. Both devices collect and digitize EEG signals from a single strip placed across the forehead. However, the algorithms of the two devices are different [21].

(A) BIS

In addition to mean and variance of the amplitude and power spectrum, higher order statistics include bispectrum for assessing EEG. The bispectrum measures the correlation of phase between different frequency components. Bispectral analysis fast and slow synchronization, calculated from a logarithm of quotient between the sum of all bispectrum peaks in band from 0.5 to $47 \mathrm{~Hz}$, and the sum of bispectrum in band from 40 to $47 \mathrm{~Hz}$, is a sub-parameter for BIS. Other sub-parameters include suppression ratios and beta relative power. A BIS number is obtained from weighted sum of the sub-parameters and a statistical multivariate model using a non-linear function. The BIS index ranges from 0 (isoelectric EEG) to 100 (awake). The exact BIS algorithm is not disclosed.

(B) Entropy

The EMG signals generally has a higher frequency than the EEG signal. Signals ranges from $0.8 \mathrm{~Hz}$ to $32 \mathrm{~Hz}$ represents EEG dominant signal and signals above $47 \mathrm{~Hz}$ are dominated by EMG. However, there is an overlap from $32 \mathrm{~Hz}$ to $47 \mathrm{~Hz}$, whether it be EEG-dominant or EMG-dominant. 
Instead of presenting the EEG values as one parameter, they are explained by $\mathrm{RE}$ of $0.8-47 \mathrm{~Hz}$ and $\mathrm{SE}$ of $0.8-32 \mathrm{~Hz}$. When " $0.8 \mathrm{~Hz}$ to $47 \mathrm{~Hz}$ " is set as the maximum (100), " $0.8 \mathrm{~Hz}$ to $32 \mathrm{~Hz}$ " naturally becomes 91 .

Entropy describes the irregularity, complexity, or unpredictability of the signal. The important equation named Shannon's entropy is as follows:

$\mathrm{E}=\sum_{1}^{n} P i \times \log \left(\frac{1}{P i}\right), P i$ indicates a probability of each frequency component, $\mathrm{n}$ indicates total number of frequency components

In the above concept, entropy is a value obtained by directly analyzing the EEG rather than the value obtained from the database as indicated by the BIS value.

\section{References}

1. Chung, H.S. Awareness and recall during general anesthesia. Korean J. Anesthesiol. 2014, 66, 339-345. [CrossRef]

2. Muncaster, A.R.; Sleigh, J.W.; Williams, M. Changes in consciousness, conceptual memory, and quantitative electroencephalographical measures during recovery from sevoflurane- and remifentanil-based anesthesia. Anesth. Analg. 2003, 96, 720-725. [CrossRef] [PubMed]

3. Sciusco, A.; Standing, J.F.; Sheng, Y.; Raimondo, P.; Cinnella, G.; Dambrosio, M. Effect of age on the performance of bispectral and entropy indices during sevoflurane pediatric anesthesia: A pharmacometric study. Paediatr. Anaesth. 2017, 27, 399-408. [CrossRef] [PubMed]

4. Saricaoglu, F; Celebi, N.; Celik, M.; Aypar, U. The evaluation of propofol dosage for anesthesia induction in children with cerebral palsy with bispectral index (BIS) monitoring. Paediatr. Anaesth. 2005, 15, 1048-1052. [CrossRef] [PubMed]

5. Choudhry, D.K.; Brenn, B.R. Bispectral index monitoring: A comparison between normal children and children with quadriplegic cerebral palsy. Anesth. Analg. 2002, 95, 1582-1585. [CrossRef]

6. Nolan, J.; Chalkiadis, G.A.; Low, J.; Olesch, C.A.; Brown, T.C. Anaesthesia and pain management in cerebral palsy. Anaesthesia 2000, 55, 32-41. [CrossRef] [PubMed]

7. Badawi, N.; Watson, L.; Petterson, B.; Blair, E.; Slee, J.; Haan, E.; Stanley, F. What constitutes cerebral palsy? Dev. Med. Child Neurol. 1998, 40, 520-527. [CrossRef] [PubMed]

8. Senbil, N.; Sonel, B.; Aydin, O.F.; Gürer, Y.K. Epileptic and non-epileptic cerebral palsy: EEG and cranial imaging findings. Brain Dev. 2002, 24, 166-169. [CrossRef]

9. Al-Sulaiman, A. Electroencephalographic findings in children with cerebral palsy: A study of 151 patients. Funct. Neurol. 2001, 16, 325-328. [PubMed]

10. Costa, V.V.; Saraiva, R.A.; Duarte, L.T. Regression of general anesthesia in patients with cerebral palsy: A comparative study using the bispectral index. Rev. Bras. Anestesiol. 2006, 56, 431-442. [CrossRef]

11. Frei, F.J.; Haemmerle, M.H.; Brunner, R.; Kern, C. Minimum alveolar concentration for halothane in children with cerebral palsy and severe mental retardation. Anaesthesia 1997, 52, 1056-1060. [CrossRef] [PubMed]

12. Sarner, J.B.; Levine, M.; Davis, P.J.; Lerman, J.; Cook, D.R.; Motoyama, E.K. Clinical characteristics of sevoflurane in children. A comparison with halothane. Anesthesiology 1995, 82, 38-46. [CrossRef] [PubMed]

13. Black, A.; Sury, M.R.; Hemington, L.; Howard, R.; Mackersie, A.; Hatch, D.J. A comparison of the induction characteristics of sevoflurane and halothane in children. Anaesthesia 1996, 51, 539-542. [CrossRef]

14. Gao, J.D.; Zhao, Y.J.; Xu, C.S.; Zhao, J.; Huang, Y.G.; Wang, T.L.; Pei, L.; Wang, J.; Yao, L.N.; Ding, Q.; et al. Evaluation of entropy for monitoring the depth of anesthesia compared with bispectral index: A multicenter clinical trial. Chin. Med. J. (Engl.) 2012, 125, 1389-1392. [PubMed]

15. Ellerkmann, R.K.; Liermann, V.M.; Alves, T.M.; Wenningmann, I.; Kreuer, S.; Wilhelm, W.; Roepcke, H.; Hoeft, A.; Bruhn, J. Spectral entropy and bispectral index as measures of the electroencephalographic effects of sevoflurane. Anesthesiology 2004, 101, 1275-1282. [CrossRef]

16. Klockars, J.G.; Hiller, A.; Münte, S.; van Gils, M.J.; Taivainen, T. Spectral entropy as a measure of hypnosis and hypnotic drug effect of total intravenous anesthesia in children during slow induction and maintenance. Anesthesiology 2012, 116, 340-351. [CrossRef] [PubMed]

17. Pilge, S.; Kreuzer, M.; Karatchiviev, V.; Kochs, E.F.; Malcharek, M.; Schneider, G. Differences between state entropy and bispectral index during analysis of identical electroencephalogram signals: A comparison with two randomised anaesthetic techniques. Eur. J. Anaesthesiol. 2015, 32, 354-365. [CrossRef] 
18. Kułak, W.; Sobaniec, W. Spectral analysis and EEG coherence in children with cerebral palsy: Spastic diplegia. Przegl. Lek. 2003, 60, 23-27. [PubMed]

19. Lysakowski, C.; Elia, N.; Czarnetzki, C.; Dumont, L.; Haller, G.; Combescure, C.; Tramèr, M.R. Bispectral and spectral entropy indices at propofol-induced loss of consciousness in young and elderly patients. Br. J. Anaesth. 2009, 103, 387-393. [CrossRef]

20. Kim, Y.S.; Chung, D.; Oh, S.K.; Won, Y.J.; Lee, I.O. Unusual elevation in Entropy but not in PSI during general anesthesia: A case report. BMC Anesthesiol. 2018, 18, 22. [CrossRef]

21. Cascella, M. Mechanisms underlying brain monitoring during anesthesia: Limitations, possible improvements, and perspectives. Korean J. Anesthesiol. 2016, 69, 113-120. [CrossRef] [PubMed]

C 2019 by the authors. Licensee MDPI, Basel, Switzerland. This article is an open access article distributed under the terms and conditions of the Creative Commons Attribution (CC BY) license (http://creativecommons.org/licenses/by/4.0/). 